\title{
Scalable Semi-Supervised Query Classification Using Matrix Sketching
}

\author{
Young-Bum Kim ${ }^{\dagger} \quad$ Karl Stratos $^{\ddagger} \quad$ Ruhi Sarikaya $^{\dagger}$ \\ ${ }^{\dagger}$ Microsoft Corporation, Redmond, WA \\ ${ }^{\ddagger}$ Columbia University, New York, NY \\ \{ybkim, ruhi.sarikaya\}@microsoft.com \\ stratos@cs.columbia.edu
}

\begin{abstract}
The enormous scale of unlabeled text available today necessitates scalable schemes for representation learning in natural language processing. For instance, in this paper we are interested in classifying the intent of a user query. While our labeled data is quite limited, we have access to virtually an unlimited amount of unlabeled queries, which could be used to induce useful representations: for instance by principal component analysis (PCA). However, it is prohibitive to even store the data in memory due to its sheer size, let alone apply conventional batch algorithms. In this work, we apply the recently proposed matrix sketching algorithm to entirely obviate the problem with scalability (Liberty, 2013). This algorithm approximates the data within a specified memory bound while preserving the covariance structure necessary for PCA. Using matrix sketching, we significantly improve the user intent classification accuracy by leveraging large amounts of unlabeled queries.
\end{abstract}

\section{Introduction}

The large amount of high quality unlabeled data available today provides an opportunity to improve performance in tasks with limited supervision through a semi-supervised framework: learn useful representations from the unlabeled data and use them to augment supervised models. Unfortunately, conventional exact methods are no longer feasible on such data due to scalability is- sues. Even algorithms that are considered relatively scalable (e.g., the Lanczos algorithm (Cullum and Willoughby, 2002) for computing eigenvalue decomposition of large sparse matrices) fall apart in this scenario, since the data cannot be stored in the memory of a single machine. Consequently, approximate methods are needed.

In this paper, we are interested in improving the performance for sentence classification task by leveraging unlabeled data. For this task, supervision is precious but the amount of unlabeled sentences is essentially unlimited. We aim to learn sentence representations from as many unlabeled queries as possible via principal component analysis (PCA): specifically, learn a projection matrix for embedding a bag-of-words vector into a lowdimensional dense feature vector. However, it is not clear how we can compute an effective PCA when we are unable to even store the data in the memory.

Recently, Liberty (2013) proposed a scheme, called matrix sketching, for approximating a matrix while preserving its covariance structure. This algorithm, given a memory budget, deterministically processes a stream of data points while never exceeding the memory bound. It does so by occasionally computing singular value decomposition (SVD) on a small matrix. Importantly, the algorithm has a theoretical guarantee on the accuracy of the approximated matrix in terms of its covariance structure, which is the key quantity in PCA calculation.

We propose to combine the matrix sketching algorithm with random hashing to completely remove limitations on data sizes. In experiments, we significantly improve the intent classification accuracy by learning sentence representations from 
huge amounts of unlabeled sentences, outperforming a strong baseline based on word embeddings trained on 840 billion tokens (Pennington et al., 2014).

\section{Deterministic Matrix Sketching}

PCA is typically performed to reduce the dimension of each data point. Let $X \in \mathbb{R}^{n \times d}$ be a data matrix whose $n$ rows correspond to $n$ data points in $\mathbb{R}^{d}$. For simplicity, assume that $X$ is preprocessed to have zero column means. The key quantity in PCA is the empirical covariance matrix $X^{\top} X \in \mathbb{R}^{d \times d}$ (up to harmless scaling). It is well-known that the $m$ length-normalized eigenvectors $u_{1} \ldots u_{m} \in \mathbb{R}^{d}$ of $X^{\top} X$ corresponding to the largest eigenvalues are orthogonal directions along which the variance of the data is maximized. Then if $\Pi \in \mathbb{R}^{d \times m}$ be a matrix whose $i$-th column is $u_{i}$, the PCA representation of $X$ is given by $X \Pi$. PCA has been a workhorse in representation learning, e.g., inducing features for face recognition (Turk et al., 1991).

Frequently, however, the number of samples $n$ is simply too large to work with. As $n$ tends to billions and trillions, storing the entire matrix $X$ in memory is practically impossible. Processing large datasets often require larger memory than the capacity of a typical single enterprise server. Clusters may enable a aggregating many boxes of memory on different machines, to build distributed memory systems achieving large memory capacity. However, building and maintaining these industry grade clusters is not trivial and thus not accessible to everyone. It is critical to have techniques that can process large data within a limited memory budget available in most typical enterprise servers.

One solution is to approximate the matrix with some $Y \in \mathbb{R}^{l \times d}$ where $l \ll n$. Many matrix approximation techniques have been proposed, such as random projection (Papadimitriou et al., 1998; Vempala, 2005), sampling (Drineas and Kannan, 2003; Rudelson and Vershynin, 2007; Kim and Snyder, 2013; Kim et al., 2015b), and hashing (Weinberger et al., 2009). Most of these techniques involve randomness, which can be undesirable in certain situations (e.g., when experiments need to be exactly reproducible). Moreover, many are not designed directly for the objective that we care about: namely, ensuring that the covariance matrices $X^{\top} X$ and $Y^{\top} Y$ remain "similar".
Input: data stream $x_{1} \ldots x_{n} \in \mathbb{R}^{d}$, sketch size $l$

1. Initialize zero-valued $Y \in 0^{l \times d}$.

2. For $i=1 \ldots n$,

(a) Insert $x_{i}$ to the first zero-valued row of $Y$.

(b) If $Y$ has no zero-valued row,

i. Compute SVD of $Y=U \Sigma V^{\top}$ where $\Sigma=$ $\operatorname{diag}\left(\sigma_{1} \ldots \sigma_{l}\right)$ with $\sigma_{1} \geq \cdots \geq \sigma_{l}$.

ii. Compute a diagonal matrix $\bar{\Sigma}$ with at least $\lceil l / 2\rceil$ zeros by setting

$$
\bar{\Sigma}_{j, j}=\sqrt{\max \left(\Sigma_{j, j}^{2}-\sigma_{\lfloor l / 2\rfloor}^{2}, 0\right)}
$$

iii. Set $Y=\bar{\Sigma} V^{\top}$.

Output: $Y \in \mathbb{R}^{l \times d}$ s.t. $\left\|X^{\top} X-Y^{\top} Y\right\|_{2} \leq 2\|X\|_{F}^{2} / l$

Figure 1: Matrix sketching algorithm by Liberty (2013). In the output, $X \in \mathbb{R}^{n \times d}$ denotes the data matrix with rows $x_{1} \ldots x_{n}$.

A recent result by Liberty (2013) gives a deterministic matrix sketching algorithm that tightly preserves the covariance structure needed for PCA. Specifically, given a sketch size $l$, the algorithm computes $Y \in \mathbb{R}^{l \times d}$ such that

$$
\left\|X^{\top} X-Y^{\top} Y\right\|_{2} \leq 2\|X\|_{F}^{2} / l
$$

This result guarantees that the error decreases in $O(1 / l)$; in contrast, other approximation techniques have a significantly worse convergence bound of $O(1 / \sqrt{l})$.

The algorithm is pleasantly simple and is given in Figure 1 for completeness. It processes one data point at a time to update the sketch $Y$ in an online fashion. Once the sketch is "full", its SVD is computed and the rows that fall below a threshold given by the median singular value are eliminated. This operation ensures that every time SVD is performed at least a half of the rows are discarded. Consequently, we perform no more than $O(2 n / l)$ SVDs on a small matrix $Y \in \mathbb{R}^{l \times d}$. The analysis of the bound (1) is an extension of the "median trick" for count sketching and is also surprisingly elementary; we refer to Liberty (2013) for details.

\section{Matrix Sketching for Sentence Representations}

Our goal is to leverage enormous quantities of unlabeled sentences to augment supervised training 
for intent classification. We do so by learning a PCA projection matrix $\Pi$ from the unlabeled data and applying it on both training and test sentences. The matrix sketching algorithm in Figure 1 enables us to compute $\Pi$ on arbitrarily large data.

There are many design considerations for using the sketching algorithm for our task.

\subsection{Original sentence representations}

We use a bag-of-words vector to represent a sentence. Specifically, each sentence is a $d$ dimensional vector $x \in \mathbb{R}^{d}$ where $d$ is the size of the vocabulary and $x_{i}$ is the count of an $n$-gram $i$ in the sentence (we use up to $n=3$ in experiments); we denote this representation by SENT. In experiments, we also use a modification of this representation, denoted by SENT+, in which we explicitly define features over the first two words in a query and also use intent predictions made by a supervised model.

\subsection{Random hashing}

When we process an enormous corpus, it can be computationally expensive just to obtain the vocabulary size $d$ in the corpus. We propose using random hashing to avoid this problem. Specifically, we pre-define the hash size $H$ we want, and then on encountering any word $w$ we map $w \rightarrow\{1 \ldots H\}$ using a fixed hash function. This allows us to compute a bag-of-words vector for any sentence without knowing the vocabulary size. See Weinberger et al. (2009) for a justification of the hashing trick for kernel methods (applicable in our setting since PCA has a kernel (dual) interpretation).

\subsection{Parallelization}

The sketching algorithm works in a sequential manner, processing each sentence at a time. While it leaves a small memory footprint, it can take prohibitively long time to process a large corpus. Liberty (2013) shows it is trivial to parallelize the algorithm: one can compute several sketches in parallel and then sketch the conjoined sketches. The theory guarantees that such layered sketches does not degrade the bound (1). We implement this parallelization to obtain an order of magnitude speedup.

\subsection{Final sentence representation:}

Once we learn a PCA projection matrix $\Pi$, we use it in both training and test times to obtain a dense feature vector of a bag-of-words sentence representation. Specifically, if $x$ is the original bag-ofwords sentence vector, the new representation is given by

$$
x_{\text {new }}=\frac{x}{\|x\|} \oplus \frac{x \Pi}{\|x \Pi\|}
$$

where $\oplus$ is the vector concatenation operation. This representational scheme is shown to be effective in previous work (e.g., see Stratos and Collins (2015)).

\subsection{Experiment}

To test our proposed method, we conduct intent classification experiments (Hakkani-Tür et al., 2013; Celikyilmaz et al., 2011; Ji et al., 2014; El-Kahky et al., 2014; Chen et al., 2016) across a suite of 22 domains shown in Table 1. An intent is defined as the type of content the user is seeking. This task is part of the spoken language understanding problem ( $\mathrm{Li}$ et al., 2009; Tur and De Mori, 2011; Kim et al., 2015c; Mesnil et al., 2015; Kim et al., 2015a; Xu and Sarikaya, 2014; Kim et al., 2015b; Kim et al., 2015d).

The amount of training data we used ranges from $12 \mathrm{k}$ to $120 \mathrm{k}$ (in number of queries) across different domains, the test data was from $2 \mathrm{k}$ to 20k. The number of intents ranges from 5 to 39 per domains. To learn a PCA projection matrix from the unlabeled data, we collected around 17 billion unlabeled queries from search logs, which give the original data matrix whose columns are bag-of- $n$-grams vector (up to trigrams) and has dimensions approximately 17 billions by 41 billions, more specifically,

$$
X \in \mathbb{R}^{17,032,086,719 \times 40,986,835,008}
$$

We use a much smaller sketching matrix $Y \in$ $\mathbb{R}^{1,000,000 \times 1,000,000}$ to approximate $X$. Note that column size is hashing size. We parallelized the sketching computation over 1,000 machines; we will call the number of machines parallelized over "batch". In all our experiments, we train a linear multi-class SVM (Crammer and Singer, 2002).

\subsection{Results of Intent Classification Task}

Table 1 shows the performance of intent classification across domains. For the baseline, SVM without embedding (w/o Embed) achieved $91.99 \%$ accuracy, which is already very competitive. However, the models with word embedding trained on 


\begin{tabular}{|c|c|cccc|}
\hline & w/o Embed & 6B-50d & $840 \mathrm{~B}-300 \mathrm{~d}$ & SENT & SENT+ \\
\hline \hline alarm & 97.25 & 97.68 & 97.5 & 97.68 & 97.74 \\
apps & 89.16 & 91.07 & 92.52 & 94.24 & 94.3 \\
calendar & 91.34 & 92.43 & 92.32 & 92.53 & 92.43 \\
communication & 99.1 & 99.13 & 99.08 & 99.08 & 99.12 \\
finance & 90.44 & 90.84 & 90.72 & 90.76 & 90.82 \\
flights & 94.19 & 92.99 & 93.99 & 94.59 & 94.59 \\
games & 90.16 & 91.79 & 92.09 & 93.08 & 92.92 \\
hotel & 93.23 & 94.21 & 93.97 & 94.7 & 94.78 \\
livemovie & 90.88 & 92.64 & 92.8 & 93.28 & 93.37 \\
livetv & 83.14 & 85.02 & 84.67 & 85.41 & 85.86 \\
movies & 93.27 & 94.01 & 93.97 & 94.75 & 95.16 \\
music & 87.87 & 90.37 & 90.9 & 91.75 & 91.33 \\
mystuff & 94.2 & 94.4 & 94.51 & 94.51 & 94.95 \\
note & 97.62 & 98.36 & 98.36 & 98.49 & 98.52 \\
ondevice & 97.51 & 97.77 & 97.6 & 97.77 & 97.84 \\
places & 97.29 & 97.68 & 97.68 & 98.01 & 97.75 \\
reminder & 98.72 & 98.96 & 98.94 & 98.96 & 98.96 \\
sports & 76.96 & 78.53 & 78.38 & 78.7 & 79.44 \\
timer & 91.1 & 91.79 & 91.33 & 92.33 & 92.61 \\
travel & 81.58 & 82.57 & 82.43 & 83.64 & 82.81 \\
tv & 91.42 & 94.11 & 94.91 & 95.19 & 95.47 \\
weather & 97.31 & 97.33 & 97.4 & 97.4 & 97.47 \\
\hline Average & 91.99 & 92.89 & 93.00 & 93.49 & 93.56 \\
\hline
\end{tabular}

Table 1: Performance comparison between different embeddings style.

6 billion tokens (6B-50d) and 840 billion tokens (840B-300d) (Pennington et al., 2014) achieved $92.89 \%$ and $93.00 \%$, respectively. 50d and $300 \mathrm{~d}$ denote size of embedding dimension. To use word embeddings as a sentence representation, we simply use averaged word vectors over a sentence, normalized and conjoined with the original representation as in (2). Surprisingly, when we use sentence representation (SENT) induced from the sketching method with our data set, we can boost the performance up to $93.49 \%$, corresponding to a $18.78 \%$ decrease in error relative to a SVM without representation. Also, we see that the extended sentence representation (SENT+) can get additional gains.

As in Table 2, we also measured performance of our method (SENT+) as a function of the percentage of unlabeled data we used from total unlabeled sentences. The overall trend is clear: as the number of sentences are added to the data for inducing sentence representation, the test performance improves because of both better coverage and better quality of embedding. We believe that if we consume more data, we can boost up the per- formance even more.

\subsection{Results of Parallelization}

Table 3 shows the sketching results for various batch size. To evaluate parallelization, we first randomly generate a matrix $\mathbb{R}^{1,000,000 \times 100}$ and it is sketched to $\mathbb{R}^{100 \times 100}$. And then we sketch run with different batch size. The results show that as the number of batch increases, we can speed up dramatically, keeping residual value $\left\|X^{\top} X-Y^{\top} Y\right\|_{2}$. It indeed satisfies the bound value, $\|X\|_{F}^{2} / l$, which was 100014503.16 .

\section{Conclusion}

We introduced how to use matrix sketching algorithm of (Liberty, 2013) for scalable semisupervised sentence classification. This algorithm approximates the data within a specified memory bound while preserving the covariance structure necessary for PCA. Using matrix sketching, we significantly improved the classification accuracy by leveraging very large amounts of unlabeled sentences. 


\begin{tabular}{|c|ccccccccccc|}
\hline & $\mathbf{0}$ & $\mathbf{1 0 \%}$ & $\mathbf{2 0 \%}$ & $\mathbf{3 0 \%}$ & $\mathbf{4 0 \%}$ & $\mathbf{5 0 \%}$ & $\mathbf{6 0 \%}$ & $\mathbf{7 0 \%}$ & $\mathbf{8 0 \%}$ & $\mathbf{9 0 \%}$ & $\mathbf{1 0 0 \%}$ \\
\hline \hline apps & 89.16 & 89.83 & 90.04 & 90.26 & 90.88 & 91.9 & 92.41 & 92.41 & 92.95 & 93.72 & 94.3 \\
music & 87.87 & 89.12 & 89.61 & 90.4 & 90.83 & 91.26 & 91.31 & 91.33 & 91.38 & 91.33 & 91.33 \\
tv & 91.42 & 92.28 & 92.83 & 93.61 & 93.96 & 94.67 & 94.91 & 95.12 & 95.34 & 95.44 & 95.47 \\
\hline
\end{tabular}

Table 2: Performance for selected domains as the number of unlabeled data increases.

\begin{tabular}{|c|c|c|}
\hline Batch Size & $\left\|X^{\mid} X-Y^{\mid} Y\right\|_{2}$ & time \\
\hline 1 & 1019779.69 & 100.21 \\
2 & 1019758.22 & 50.31 \\
4 & 1019714.19 & 26.50 \\
5 & 1019713.43 & 21.67 \\
8 & 1019679.67 & 14.53 \\
10 & 1019692.67 & 12.13 \\
16 & 1019686.35 & 8.53 \\
20 & 1019709.03 & 7.35 \\
25 & 1019650.51 & 6.40 \\
40 & 1019703.24 & 4.97 \\
50 & 1019689.33 & 4.48 \\
\hline
\end{tabular}

Table 3: Results for corresponding batch size. Second column indicates the norm of gap between original and sketching matrix. Time represents the running time for sketching methods, measured in seconds.

\section{References}

Asli Celikyilmaz, Dilek Hakkani-Tür, and Gokhan Tür. 2011. Leveraging web query logs to learn user intent via bayesian discrete latent variable model. ICML.

Yun-Nung Chen, Dilek Hakkani-Tür, and Xiaodong He. 2016. Zero-shot learning of intent embeddings for expansion by convolutional deep structured semantic models. In Proc. of ICASSP.

Koby Crammer and Yoram Singer. 2002. On the learnability and design of output codes for multiclass problems. Machine Learning, 47(2-3):201-233.

Jane K Cullum and Ralph A Willoughby. 2002. Lanczos Algorithms for Large Symmetric Eigenvalue Computations: Vol. 1: Theory, volume 41. SIAM.

Petros Drineas and Ravi Kannan. 2003. Pass efficient algorithms for approximating large matrices. In SODA, volume 3, pages 223-232.

Ali El-Kahky, Xiaohu Liu, Ruhi Sarikaya, Gokhan Tur, Dilek Hakkani-Tur, and Larry Heck. 2014. Extending domain coverage of language understanding systems via intent transfer between domains using knowledge graphs and search query click logs. In Acoustics, Speech and Signal Processing (ICASSP), 2014 IEEE International Conference on, pages 4067-4071. IEEE.
Dilek Hakkani-Tür, Asli Celikyilmaz, Larry P Heck, and Gökhan Tür. 2013. A weakly-supervised approach for discovering new user intents from search query logs. In INTERSPEECH, pages 3780-3784.

Yangfeng Ji, Dilek Hakkani-Tur, Asli Celikyilmaz, Larry Heck, and Gokhan Tur. 2014. A variational bayesian model for user intent detection. In Acoustics, Speech and Signal Processing (ICASSP), 2014 IEEE International Conference on, pages 40724076. IEEE.

Young-Bum Kim and Benjamin Snyder. 2013. Optimal data set selection: An application to graphemeto-phoneme conversion. In HLT-NAACL, pages 1196-1205.

Young-Bum Kim, Minwoo Jeong, Karl Stratos, and Ruhi Sarikaya. 2015a. Weakly supervised slot tagging with partially labeled sequences from web search click logs. In Proc. of the Conference on the North American Chapter of the Association for Computational Linguistics - Human Language Technologies, pages 84-92.

Young-Bum Kim, Karl Stratos, Xiaohu Liu, and Ruhi Sarikaya. 2015b. Compact lexicon selection with spectral methods. In Proc. of Annual Meeting of the Association for Computational Linguistics: Human Language Technologies.

Young-Bum Kim, Karl Stratos, and Ruhi Sarikaya. 2015c. Pre-training of hidden-unit crfs. In Proc. of Annual Meeting of the Association for Computational Linguistics: Human Language Technologies, pages 192-198.

Young-Bum Kim, Karl Stratos, Ruhi Sarikaya, and Minwoo Jeong. 2015d. New transfer learning techniques for disparate label sets. In Proc. of Annual Meeting of the Association for Computational Linguistics: Human Language Technologies.

Xiao Li, Ye-Yi Wang, and Alex Acero. 2009. Extracting structured information from user queries with semi-supervised conditional random fields. In Proceedings of the 32nd international ACM SIGIR conference on Research and development in information retrieval.

Edo Liberty. 2013. Simple and deterministic matrix sketching. In Proceedings of the 19th ACM SIGKDD international conference on Knowledge discovery and data mining, pages 581-588. ACM. 
Grégoire Mesnil, Yann Dauphin, Kaisheng Yao, Yoshua Bengio, Li Deng, Dilek Hakkani-Tur, Xiaodong He, Larry Heck, Gokhan Tur, Dong Yu, et al. 2015. Using recurrent neural networks for slot filling in spoken language understanding. $A u$ dio, Speech, and Language Processing, IEEE/ACM Transactions on, 23(3):530-539.

Christos H Papadimitriou, Hisao Tamaki, Prabhakar Raghavan, and Santosh Vempala. 1998. Latent semantic indexing: A probabilistic analysis. In Proceedings of the seventeenth ACM SIGACT-SIGMOD-SIGART symposium on Principles of database systems, pages 159-168. ACM.

Jeffrey Pennington, Richard Socher, and Christopher D Manning. 2014. Glove: Global vectors for word representation. Proceedings of the Empiricial Methods in Natural Language Processing (EMNLP 2014), 12:1532-1543.

Mark Rudelson and Roman Vershynin. 2007. Sampling from large matrices: An approach through geometric functional analysis. Journal of the ACM (JACM), 54(4):21.

Karl Stratos and Michael Collins. 2015. Simple semisupervised pos tagging. In Proceedings of NAACLHLT, pages 79-87.

Gokhan Tur and Renato De Mori. 2011. Spoken language understanding: Systems for extracting semantic information from speech. John Wiley \& Sons.

Matthew Turk, Alex P Pentland, et al. 1991. Face recognition using eigenfaces. In Computer Vision and Pattern Recognition, 1991. Proceedings CVPR'91., IEEE Computer Society Conference on, pages 586-591. IEEE.

Santosh S Vempala. 2005. The random projection method, volume 65. American Mathematical Soc.

Kilian Weinberger, Anirban Dasgupta, John Langford, Alex Smola, and Josh Attenberg. 2009. Feature hashing for large scale multitask learning. In Proceedings of the 26th Annual International Conference on Machine Learning, pages 1113-1120. ACM.

Puyang Xu and Ruhi Sarikaya. 2014. Contextual domain classification in spoken language understanding systems using recurrent neural network. In Acoustics, Speech and Signal Processing (ICASSP), 2014 IEEE International Conference on, pages 136140. IEEE. 